Virginia Commonwealth University VCU Scholars Compass

2011

\title{
Sc-phthalocyanine sheet: Promising material for hydrogen storage
}

\author{
Kun Lü \\ Jian Zhou \\ College of Engineering \\ Le Zhou \\ College of Engineering \\ See next page for additional authors
}

Center for Applied Physics and Technology

Follow this and additional works at: http://scholarscompass.vcu.edu/phys_pubs

Part of the Physics Commons

Lu, K., Zhou, J., Zhou, L., et al. Sc-phthalocyanine sheet: Promising material for hydrogen storage. Applied Physics Letters, 99, 163104 (2011). Copyright (C) 2011 AIP Publishing LLC.

\section{Downloaded from}

http://scholarscompass.vcu.edu/phys_pubs/28

This Article is brought to you for free and open access by the Dept. of Physics at VCU Scholars Compass. It has been accepted for inclusion in Physics Publications by an authorized administrator of VCU Scholars Compass. For more information, please contact libcompass@vcu.edu. 
Authors

Kun Lü, Jian Zhou, Le Zhou, Qian Wang, Qiang Sun, and Puru Jena 


\title{
Sc-phthalocyanine sheet: Promising material for hydrogen storage
}

\author{
Kun Lü, ${ }^{1}$ Jian Zhou, ${ }^{2}$ Le Zhou, ${ }^{2}$ Qian Wang, ${ }^{1,3}$ Qiang Sun,,${ }^{1,2,3, a)}$ and Puru Jena ${ }^{3}$ \\ ${ }^{1}$ Center for Applied Physics and Technology, Peking University, Beijing 100871, China \\ ${ }^{2}$ Department of Materials Science and Engineering, College of Engineering, Peking University, \\ Beijing 100871, China \\ ${ }^{3}$ Department of Physics, Virginia Commonwealth University, Richmond, Virginia 23284, USA
}

(Received 24 August 2011; accepted 28 September 2011; published online 18 October 2011)

\begin{abstract}
It has been a long-standing dream to have high surface area materials with isolated and exposed transition-metal ions for hydrogen storage. The flexible synthesis procedure proposed recently by M. Abel, et al. [J. Am. Chem. Soc. 133, 1203 (2011)] and A. Sperl et al. [J. Am. Chem. Soc. 133, 11007 (2011)] provides a different pathway to achieve this goal. Using first-principles theory and grand canonical Monte Carlo simulation, we carry out a systematic study of $3 \mathrm{~d}$ transition metals ( $\mathrm{Sc}$ to $\mathrm{Zn}$ )-phthalocyanine porous sheets and find that Sc-phthalocyanine can store $4.6 \mathrm{wt}$. \% hydrogen at $298 \mathrm{~K}$ and 100 bar. (ㅇ 2011 American Institute of Physics. [doi:10.1063/1.3653465]
\end{abstract}

The biggest challenge to a hydrogen economy is to find materials that can store hydrogen with sufficient gravimetric and volumetric density at ambient temperature and pressure to meet the requirements of the transportation industry. No such materials exist at this time. ${ }^{1}$ Materials that meet the density requirements do not meet the thermodynamic requirements and vise versa. For example, light metal hydrides can store hydrogen with gravimetric density as high as $20 \mathrm{wt} . \%$, but hydrogen storage is not reversible and it requires very high temperature for hydrogen desorption. Materials such as carbon nanotubes, metal and covalent organic frameworks (MOFs, COFs) can store hydrogen reversibly, but hydrogen desorbs at very low temperature. This is because, in light metal hydrides, hydrogen is held in rather strong covalent or ionic bonds, while in MOFs, hydrogen is weakly bound. It is ideal to have materials where hydrogen is bound with binding energy intermediate between physisorption and chemisorption. Two such mechanisms have been proposed earlier, one by Kubas and the other by Jena and coworkers. In the Kubas mechanism, ${ }^{2-4}$ hydrogen binds to transition metal (TM) atom in quasi-molecular form through charge donation from hydrogen to the unfilled d-orbitals of the metal atom and back donation from metal atom to the antibonding orbital of the hydrogen molecule. In the mechanism proposed by Jena and coworkers, ${ }^{5-8}$ hydrogen binds to metal cations through a charge polarization mechanism where the electric field produced by the metal ion polarizes the hydrogen molecule. Typical binding energies associated with lone transition metal atoms and the Kubas mechanism are about $0.5 \mathrm{eV} / \mathrm{H}_{2}$, while that associated with lone non transition metal ions and the charge polarization mechanism are about $0.2 \mathrm{eV} / \mathrm{H}_{2}$. The difficulty is that transition metal atoms doped on substrates have a tendency to coalesce ${ }^{9}$ while binding of $\mathrm{H}_{2}$ molecules on light metal cations such as $\mathrm{Li}$ is lower than the desired value. ${ }^{10}$ What would be ideal is to have a material composed of light elements such as $\mathrm{C}$ where doped transition metal atoms can be well dispersed and prevented from coalescence. Such materials have been scarce.

\footnotetext{
${ }^{\text {a) }}$ Author to whom correspondence should be addressed. Electronic mail: sunqiang@pku.edu.cn.
}

In a recent paper, Abel et al. ${ }^{11}$ have developed a synthesis technique where $\mathrm{Fe}$-phthalocyanine $(\mathrm{FePc})$ forms a periodic single layer sheet with regularly spaced $\mathrm{Fe}$ atoms. The synthesis procedure is flexible so that $\mathrm{Fe}$ atoms can be replaced by other metal atoms. ${ }^{11,12}$ The question is: Can Fe atoms in FePc trap hydrogen effectively? Are other transition metals better suited for the purpose? We have explored the potential of these stable organo-metallic porous sheets with regularly and separately exposed TM atoms to naturally store hydrogen. In this communication, we present our results based on multiscale modeling that combines first-principles density functional theory and grand canonical Monte Carlo (GCMC) simulation. We show that Sc-phthalocyanine $(\mathrm{ScPc})$ has all the desirable features as a hydrogen storage material.

We used the density functional theory with PerdewBurke-Enzerhof $(\mathrm{PBE})^{13}$ form for exchange correlation potential and the projector augmented wave (PAW) method $^{14}$ implemented in the Vienna ab initio simulation

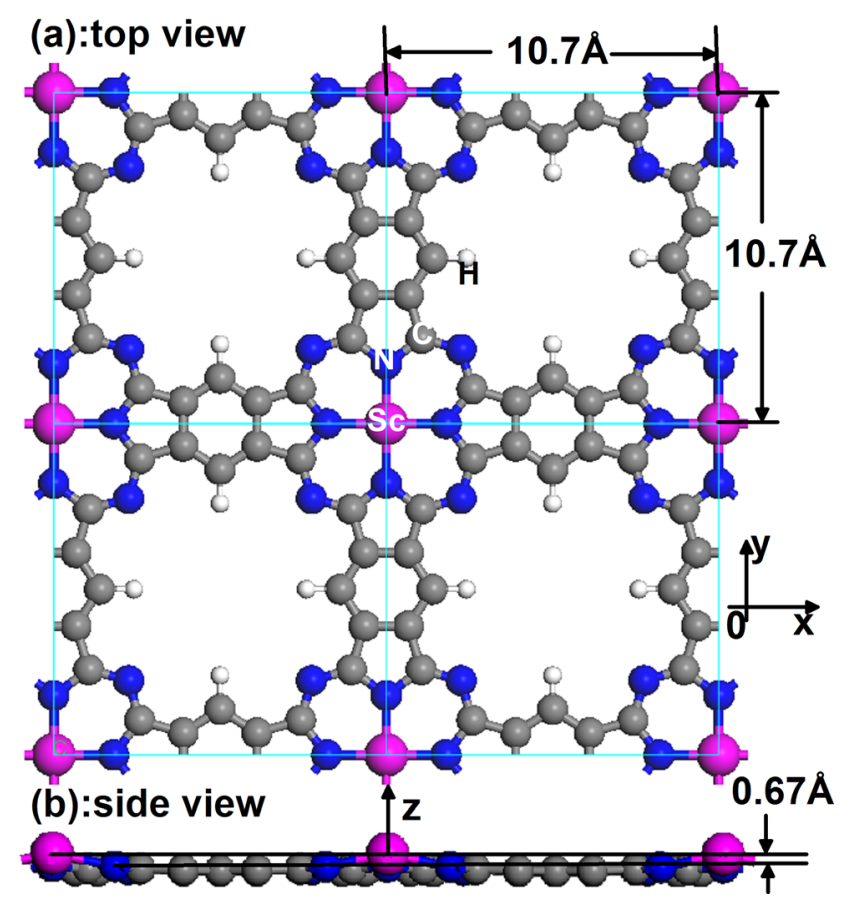

FIG. 1. (Color online) Geometry of Sc-phthalocyanine sheet. 

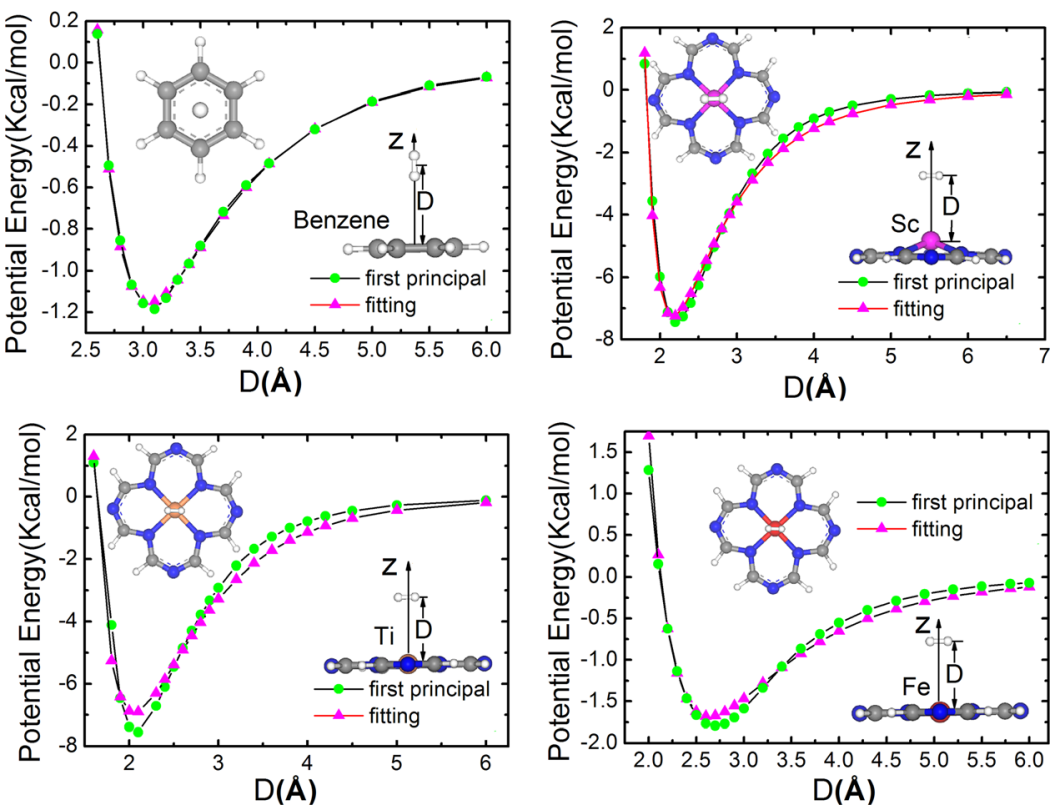

FIG. 2. (Color online) Potential energy curves of $\mathrm{H}_{2}$ on substrates derived from first-principles calculations and force field. package (VASP). ${ }^{15}$ The optimized unit cell parameters for FePc are $\mathrm{a}=\mathrm{b}=10.7 \AA$ which agree very well with the experimental values ${ }^{11}(\mathrm{a}=\mathrm{b}=10.5 \pm 0.1 \AA)$. The lattice parameters for ScPc sheet are $a=b=10.7 \AA$. Due to its larger atomic radius as compared to other first-row TMs, Sc atom is out of the plane by $0.67 \AA$, as shown in Figure 1 . To calculate the accurate adsorption energy of $\mathrm{H}_{2}$ on metal sites, we have used long range correlated hybrid functional WB97XD (Ref. 16) for exchange and correlation energy. 6-311 G(d) basis was used for $\mathrm{C}, \mathrm{H}$, and $\mathrm{N}$, and the LanL2TZ+ (Ref. 17; triple zeta basis designed for effective core potential with diffuse $\mathrm{d}$ function) basis was applied for metal atoms, as implemented in Gaussian 09 program package. ${ }^{18}$ The adsorption energy of one $\mathrm{H}_{2}$ on TMPc is found to be 5.2816, 6.6154, 1.0458, $1.2529,3.0186,1.9872,0.9923,1.0786,0.9614$, and $1.3506 \mathrm{kcal} / \mathrm{mol}$ for $\mathrm{TM}=\mathrm{Sc}, \mathrm{Ti}, \mathrm{V}, \mathrm{Cr}, \mathrm{Mn}, \mathrm{Fe}, \mathrm{Co}, \mathrm{Ni}, \mathrm{Cu}$, and $\mathrm{Zn}$, respectively. Since the optimum energy window for ideal hydrogen binding energy is $3.61-7.22 \mathrm{kcal} / \mathrm{mol}$ at room temperature, ${ }^{19}$ we note from the above that only ScPc and TiPc satisfy this requirement. Because of the recent experimental synthesis of FePc, our discussions are first extended to these three systems.

We use the following Lennard-Jones potential to describe the interactions of $\mathrm{H}_{2}$ with the sheet,

$$
U_{i j}\left(r_{i j}\right)=4 \varepsilon\left[\left(\frac{\sigma}{r_{i j}}\right)^{12}-\left(\frac{\sigma}{r_{i j}}\right)^{6}\right],
$$

where $\varepsilon$ represents the depth of the potential well, $\sigma$ is the finite distance at which the inter-particle potential is zero, and $\mathrm{r}_{\mathrm{ij}}$ is the distance between the particles. The hydrogenhydrogen interactions were described by the DREIDING forcefield $^{20} \quad(\varepsilon=0.0152 \mathrm{kcal} / \mathrm{mol}, \sigma=2.846 \AA)$. To obtain these parameters, we used the quadruple-zeta-valence basis set plus polarization and diffuse basis function (labeled as def2-QZVPPD) ${ }^{21}$ for metal atoms, and the triple-zeta-valence basis set plus two sets of polarization and diffuse basis (labeled as def-TZVPPD) ${ }^{21}$ for other atoms. The potential curves are shown in Figure 2, and fitted parameters are listed in Table I. GCMC simulations are then performed as implemented in the MUSIC Code. ${ }^{22}$ A vacuum space of $32 \AA$ is added to the sheets. The simulation was conducted in a constant volume system defined by a simulation box consisting of $4 \times 4 \times 1$ unit cells. Hydrogen molecules were randomly inserted, moved, and deleted, which allowed the number of the total molecules, N, and configurational energy, E, to fluctuate at constant temperature and chemical potential. For each state point, $4 \times 10^{6}$ steps are used in GCMC simulations to guarantee the equilibrium followed by $6 \times 10^{6}$ steps to sample the thermodynamic properties.

The results are given in Figure 3 . At $\mathrm{T}=77 \mathrm{~K}$, the highest $\mathrm{H}_{2}$ gravimetric storage capacities are found to be near 40 bar. The corresponding hydrogen gravimetric densities are 12.5 wt. $\%, 11.0$ wt. \%, and 8.6 wt. \% for ScPc, TiPc, andFePc, respectively. These values exceed the reported hydrogen storage capacities in MOFs under similar conditions $^{23,24}$ (for example, 5.2 wt. \% for MOF-5, $7.5 \mathrm{wt}$. \% for MOF-177). At $\mathrm{T}=298 \mathrm{~K}$ and $\mathrm{P}=100 \mathrm{bar}$, the gravimetric density of $\mathrm{H}_{2}$ reaches $4.6 \mathrm{wt}$. \% for $\mathrm{ScPc}$, which is comparable to the revised 2010 DOE targets $\left(0.045 \mathrm{Kg} \mathrm{H}_{2} / \mathrm{Kg}\right.$ system, that is, $4.3 \mathrm{wt}$. $\%$ for system gravimetric capacity) for on-board hydrogen storage systems.

It is interesting to note that although $\mathrm{H}_{2}$ has larger adsorption energy on TiPc, the performance of ScPc is better. The possible reasons are the following: due to the larger size of Sc, it is out of the Pc plane and become more exposed, which reduces the steric hindrance effects when

TABLE I. Fitted potential parameters.

\begin{tabular}{lccccc}
\hline Term $\left(\mathrm{H} \_-\right)$ & $\varepsilon(\mathrm{kcal} / \mathrm{mol})$ & $\sigma(\AA)$ & Term (H_— $)$ & $\varepsilon(\mathrm{kcal} / \mathrm{mol})$ & $\sigma(\AA)$ \\
\hline H_ & 0.01520 & 2.84642 & $\mathrm{Sc}$ & 0.82388 & 1.83573 \\
H_A & 0.02806 & 3.55824 & Ti & 0.10221 & 1.72816 \\
N_A & 0.01580 & 3.40757 & Fe & 0.01041 & 2.34128 \\
N_B(Sc) & 0.44615 & 3.15637 & C_A & 0.10242 & 2.68000 \\
N_B(Ti) & 0.64229 & 2.51325 & C_B & 0.11687 & 3.12360 \\
N_B(Fe) & 0.01499 & 3.28603 & & & \\
\hline
\end{tabular}



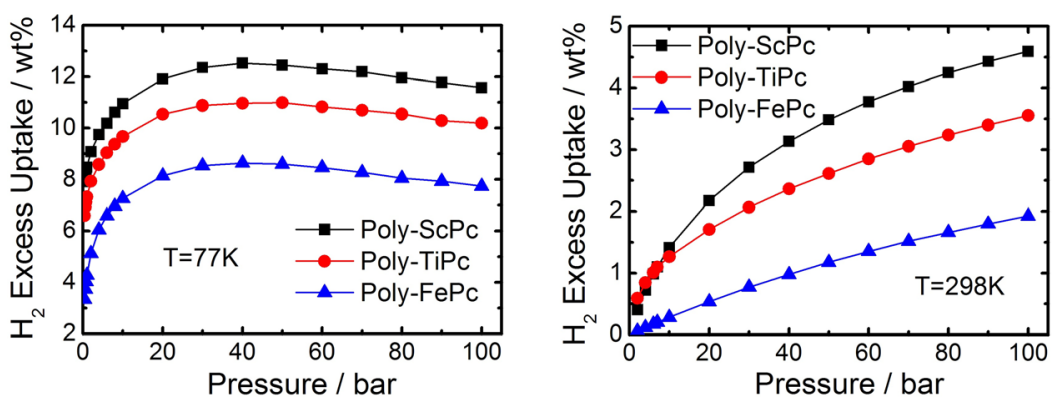

FIG. 3. (Color online) Predicted $\mathrm{H}_{2}$ excess adsorption isotherms for TMPc $(\mathrm{TM}=\mathrm{Fe}, \mathrm{Sc}, \mathrm{Ti})$ at $\mathrm{T}=77 \mathrm{~K}$ and $298 \mathrm{~K}$. more $\mathrm{H}_{2}$ molecules are introduced to the Sc site. Furthermore, the smaller mass of Sc would favor the higher weight percentage of storage.

To get further insight into the interaction between $\mathrm{H}_{2}$ and $\mathrm{ScPc}$, we performed natural bond orbital (NBO) analy$\mathrm{sis}^{25}$ where the electronic wave function is interpreted in terms of a set of occupied Lewis and a set of unoccupied non-Lewis delocalized orbitals. For each donor NBO (i) and acceptor NBO (j), the stabilization energy E(2) associated with charge transfer $\mathrm{i} \rightarrow \mathrm{j}$ is estimated as

$$
E(2)=\Delta E_{i j}=q_{i}\left[\frac{F(i, j)^{2}}{\varepsilon_{j}-\varepsilon_{i}}\right]
$$

where $\mathrm{q}_{\mathrm{i}}$ is the donor orbital occupancy, $\varepsilon_{\mathrm{i}}$ and $\varepsilon_{\mathrm{j}}$ are the diagonal elements (orbital energies), and $\mathrm{F}(\mathrm{i}, \mathrm{j})$ is the off-diagonal NBO Fock matrix element. We have identified that there are two main channels of electron donation from the bonding state of $\mathrm{H}_{2}$ to the spin-up orbital with the component of s-47.975\% and $\mathrm{d}-51.32 \%$, and to the spin-down orbital with the component of $\mathrm{s}-49.1 \%$ and $\mathrm{d}-49.9 \%$; the corresponding donation of electrons is 0.024 and 0.020 , respectively, resulting in the stabilization energy $\mathrm{E}(2)$ of 14.0 and $13.6 \mathrm{kcal} / \mathrm{mol}$. However, back donation mainly takes place between the spin-up orbital with the component of s- $0.85 \%$ and d-99.15\% to the antibonding state of $\mathrm{H}_{2}$, and about 0.011 electrons are back donated giving energy of $2.45 \mathrm{kcal} / \mathrm{mol}$ to $\mathrm{E}(2)$. We can clearly see that the interactions between $\mathrm{H}_{2}$ and $\mathrm{ScPc}$ follow the Kubas mechanism. For TiPc system, there are also two main channels for charge donations: the spin-up channel with s-46.78\% and $\mathrm{d}-44.54 \%$ and the spin-down channel with $\mathrm{s}-71.93 \%$, p-13.82\%, and d-14.25\%, contributing to E(2) with 13.96 and $16.26 \mathrm{kcal} / \mathrm{mol}$, respectively, while the electron back donation mainly takes place in the spin-down channel with the orbital components of $\mathrm{p}-99.6 \%$ and $\mathrm{d}-0.4 \%$. The corresponding $\mathrm{E}(2)$ is found to be $3.9 \mathrm{kcal} / \mathrm{mol}$. The situations are different in FePc system, the d-orbital components in the charge transfer channels are much less as compared to the former two systems. The main spin-up channel has the components of $\mathrm{s}-99.3 \%, \mathrm{p}-0.3 \%$, and $\mathrm{d}-0.3 \%$, while the spin-down channel has the components of $\mathrm{s}-4.2 \%, \mathrm{p}-88.46 \%$, and $\mathrm{d}-7.33 \%$. The corresponding contributions to $\mathrm{E}(2)$ are 4.35 and $5.55 \mathrm{kcal} /$ mol, respectively. Moreover, there is one main channel with $\mathrm{p}-100 \%$, respectively, in spin-up and spin-down for the charge back donation, and their contributions to $E(2)$ are 3.08 and $3.15 \mathrm{kcal} / \mathrm{mol}$. For TiPc and FePc systems, the numbers of donated charges are $0.195 \mathrm{e}$ and $0.042 \mathrm{e}$, the corresponding back donations are $0.016 \mathrm{e}$ and $0.002 \mathrm{e}$.
In summary, multi-scale modeling studies have been carried out to investigate the performance of TMPc porous sheets for storing hydrogen. The $\mathrm{ScPc}$ sheet is indentified as a promising hydrogen storage material, because Sc is more exposed and has a lighter mass than other $3 \mathrm{~d}$ transition metals. Detailed NBO analysis showed that Kubas mechanism is involved in this system. At temperature of $298 \mathrm{~K}$ and pressure of 100 bar, the excess $\mathrm{H}_{2}$ uptake is found to be $4.6 \mathrm{wt}$. \% which is comparable to the target set by the USA Department of Energy for 2010. We hope that the present study will stimulate further experimental effort in this system.

This work is partially supported by grants from the National Grand Fundamental Research 973 Program of China (No. 2010CB631301), the National Natural Science Foundation of China (NNSFC-10874007, 20973010, 21173007), and from the U.S. Department of Energy.

${ }^{1}$ P. Jena, J. Phys. Chem. Lett. 2, 206 (2011).

${ }^{2}$ G. J. Kubas, Acc. Chem. Res. 21, 120 (1988).

${ }^{3}$ Y. Zhao, Y. H. Kim, A. C. Dillon, J. M. Heben, and S. B. Zhang, Phys. Rev. Lett. 94, 155504 (2005).

${ }^{4}$ T. Yildrim and S. Ciraci, Phys. Rev. Lett. 94, 175501 (2005).

${ }^{5}$ B. K. Rao and P. Jena, Europhys. Lett. 20, 307 (1992).

${ }^{6}$ J. Niu, B. K. Rao, and P. Jena, Phys. Rev. Lett. 68, 2277 (1992).

${ }^{7}$ J. Niu, B. K. Rao, P. Jena, and M. Manninen, Phys. Rev. B 51, 4475 (1995).

${ }^{8}$ J. Zhou, Q. Wang, Q. Sun, P. Jena, and X. S. Chen, Proc. Natl. Acad. Sci.

U.S.A. 107, 2801 (2010).

${ }^{9}$ Q. Sun, Q. Wang, P. Jena, and Y. Kawazoe, J. Am. Chem. Soc. 127, 14582 (2005).

${ }^{10}$ Q. Sun, P. Jena, Q. Wang, and M. Marquez, J. Am. Chem. Soc. 128, 9741 (2006).

${ }^{11}$ M. Abel, S. Clair, O. Ourdjini, M. Mossoyan, and L. Porte, J. Am. Chem. Soc. 133, 1203 (2011).

${ }^{12}$ A. Sperl, J. Kroger, and R. Berndt, J. Am. Chem. Soc. 133, 11007 (2011).

${ }^{13}$ J. P. Perdew, K. Burke, and M. Ernzerhof, Phys. Rev. Lett. 77, 3865 (1996).

${ }^{14}$ P. E. Blöchl, Phys. Rev. B 20, 17953 (1994).

${ }^{15}$ G. Kresse and J. Furthmüller, Phys. Rev. B 54, 11169 (1996).

${ }^{16}$ J. D. Chai and M. Head-Gordon, Phys. Chem. Chem. Phys. 10, 6615 (2008).

${ }^{17}$ P. J. Hay and W. R. Wadt, J. Chem. Phys. 82, 270 (1985).

${ }^{18}$ M. J. Frisch, G. W. Trucks, and H. B. Schlegel, et al., Gaussian 09, Revision B.01, Gaussian, Inc., Wallingford, CT, 2010.

${ }^{19}$ S. K. Bhatia and A. L. Myers, Langmuir 22, 1688 (2006).

${ }^{20}$ S. L. Mayo, B. D. Olafson, and W. A. Goddard, J. Phys. Chem. 94, 8897 (1990).

${ }^{21}$ D. Rappoport and F. Furche, J. Chem. Phys. 133, 134105 (2010).

${ }^{22}$ A. Gupta, S. Chempath, M. Sanborn, L. Clark, and R. Snurr, Mol. Simul. 29, 29 (2003).

${ }^{23}$ A. G. Wong-Foy, A. J. Matzger, and O. M. Yaghi, J. Am. Chem. Soc. 128, 3494 (2006).

${ }^{24}$ H. Furukawa, M. A. Miller, and O. M. Yaghi, J. Mater. Chem. 17, 3197 (2007).

${ }^{25}$ A. E. Reed, L. A. Curtiss, and F. Weinhold, Chem. Rev. 88, 899 (1988). 